\title{
SOLVING ECONOMIC DISPATCH PROBLEM USING HYBRID GA-PS-SQP METHOD
}

\author{
Jamal S. Alsumait, Student Member, Jan K. Sykulski, Senior Member
}

\begin{abstract}
This study presents a new approach to solve the well-known power system Economic Load Dispatch problem (ED) using a hybrid algorithm consisting of Genetic Algorithm (GA), Pattern Search (PS) and Sequential Quadratic Programming (SQP). GA is the main optimizer of this algorithm, whereas PS and SQP are used to fine-tune the results obtained from the GA, thereby increasing solution confidence. To test the effectiveness of this approach it was applied to various test systems. Furthermore, the convergence characteristics and robustness of the proposed method have been explored through comparisons with results reported in literature. The outcome is very encouraging and suggests that the hybrid GA-PS-SQP algorithm is very effective in solving the power system economic load dispatch problem.
\end{abstract}

Index Terms: Economic Dispatch, Valve-Point effect, Pattern Search method (PS), Genetic Algorithms (GA) and Sequential Quadratic Programming (SQP).

\section{INTRODUCTION}

The optimal unit commitment allocation of the electric power system has caught the attention of researchers in recent years. Many methods were proposed to solve the economic dispatch problem (ED), which resulted in optimal power system units generation scheduling. In addition, the use of hybrid methods to achieve the optimal unit commitment allocation has been presented in the literature extensively. The combination of two or three hybrid methods to solve the ED problem has been shown to improve results and to overcome drawbacks of each method separately. In previous publications by the authors of this paper [1,2], which implemented the pattern search method (PS), the authors faced some difficulties in choosing the point with which the algorithm used to start the search. The choice of the starting point that was provided to the PS was the main weakness of the algorithm. The new hybrid method eliminates the need of PS and SQP to the initial guess. The algorithm was applied to various test systems to validate the accuracy of the results. Moreover, we compared this approach with other methods to point out the advantages of the proposed method.

In the pursuit of the optimal solution, hybrid methods have been investigated and developed by many researchers. In [3], the authors used the conventional Lagrangian relaxation approach, where the first order gradient method and multi-pass dynamic programming were combined together. Moreover, the authors stated that the proposed method has no restrictions on generator cost function, and it performs a direct search of the feasible solution at each step. The use of two hybrid techniques based on Genetic Algorithm (GA) and Simulated Annealing (SA) to evaluate the trade-off between fuel cost and environmental impact in economic dispatch was presented in [4]. The authors combined the total emission of the individual pollutants into a criterion via the use of relative weight in the main objective function. A two phase GA approach to solve the economic and emission dispatch was proposed in [5]. In the first phase the authors searched for the incremental cost factor using binary coded GA. Then the best solution of the first phase was taken as the initial condition in the second phase. The results were compared with other methods and were considered equally reliable and promising. However, the constraints for security and ramp rate were not included in the modelling of the system. In [6], the authors introduced hybrid evolutionary algorithms to solve the economic dispatch problem with valve-point effect, i.e. Particle Swarm Optimization (PSO) and Sequential Quadratic Programming (SQP). The valve-point effect explanation and definition will be presented later in this section. They used PSO as the main optimizer of the objective function and SQP as the fine tuner for every improvement in the solution of the PSO run.

Another hybrid approach with different heuristic methods was implemented in [7]. The Differential Evolution (DE) and the Sequential Quadratic Programming (SQP) were combined in to a single algorithm that is able to solve the economic dispatch problem. The algorithm was used on 13 and 40 thermal units whose incremental fuel cost function contains the valve-point loading effect. Another interesting article showing the importance of the usage of hybrid algorithms is presented in [8]. The authors combined three evolutionary methods to solve a fuzzy modelled Unit Commitment Problem (UCP). The three methods are Tabu Search (TS), Particle Swarm Optimization (PSO) and Sequential Quadratic Programming (SQP) or simply hybrid TS-PSO-SQP. TS is used to solve the combinatorial sub-problem of the UCP. Then the non-linear programming subproblem of the UCP is solved using the hybrid PSOQSP technique.

The main objective of this study is to introduce a hybrid method that combines the Genetic Algorithm (GA), Pattern Search (PS) and Sequential Quadratic Programming (SQP) - referred to as the hybrid GAPS-SQP method - in the context of power system economic load dispatch problem with a valve-point effect. The GA has been used in the primary stage to nominate an initial guess, then the hybrid PS-SQP has 
been implemented in the final stage to fine tune the results. The valve-point effect is a 'ripple' added to the generating unit's curve when each steam admission valve in a turbine starts to open. Therefore, to improve accuracy when using this model, an additional term representing the valve-point effect is added to the cost function as suggested in [9]. The addition of the valve-point effect poses a more challenging task to the proposed method since it increases the non-linearity of the search space as well as the number of local minima (see Figure 1).

The paper is organized as follows: Section 2 introduces the problem formulation; Section 3 presents a description of the proposed PS algorithm; the analysis and test results are included in Section 4, followed by concluding remarks.

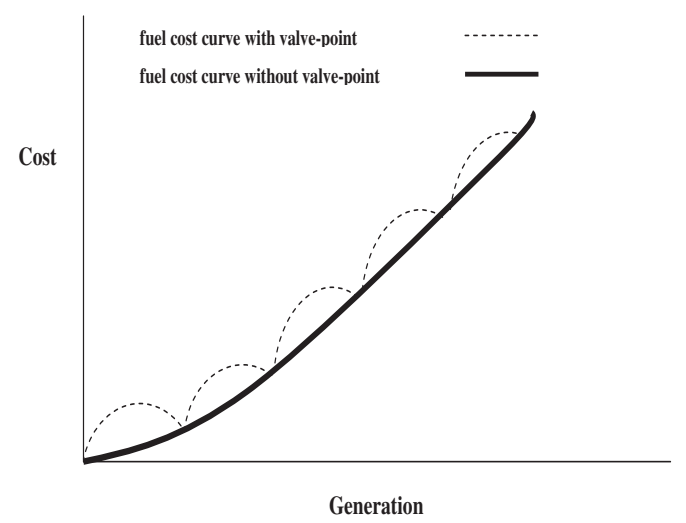

Fig.1 - The valve-point effect

\section{PROBLEM FORMULATION}

The traditional formulation of the economic load dispatch problem is a minimization of summation of the fuel costs of the individual dispatchable generators subject to the real power balanced with the total load demand as well as the limits on generators outputs. In mathematical terms the problem may be stated as:

$$
F=\sum_{i=1}^{N} F_{i}\left(P_{i}\right)
$$

The incremental fuel cost functions of the generation units with valve-point loading are represented as follows [10]:

$$
F_{i}\left(P_{i}\right)=a_{i} P_{i}^{2}+b_{i} P_{i}+c_{i}+\left|e_{i} \times \sin \left(f_{i} \times\left(P_{i \min }-P_{i}\right)\right)\right|
$$

subject to

$$
\begin{gathered}
\sum_{i=1}^{N} P_{g i}=P_{D}+P_{L} \\
P_{g i(\min )}<P_{g i}<P_{g i(\max )}, i \in N_{s}
\end{gathered}
$$

where:

$F$ is the system overall cost function

$N$ the number of generators in the system

$d_{i}, b_{i}, c_{i}$ the constants of fuel function of generator number i

$e_{i}, f_{i}$ the constants of the valve point effect of generator number $\mathrm{i}$

$P_{g i}$ the active power generation of generator number $\mathrm{i}$

$P_{D}$ the total power system demand

$P_{L}$ the total system transmission losses

$P_{g i(\min )}$ the minimum limit on active power generation of generator $\mathrm{i}$

$P_{g i(\max )}$ the maximum limit on active power generation of generator $i$

$N_{s}$ the set of generators in the system

The sinusoidal term added to the fuel cost function which models the valve-point effect creates ripples in the heat-rate curve and therefore introduces more local minima to the search space.

\section{PATTERN SEARCH METHOD}

Due to the limitations on the length of this paper, and good coverage of the GA and SQP techniques in the literature, only the PS method will be briefly mentioned here. The Pattern Search (PS) is an evolutionary routine suitable for solving a variety of optimization problems that lie outside the scope of typical optimization tasks, and has the advantage of being very simple in concept, easy to implement and computationally efficient. Unlike, say, genetic algorithms [11, 12], the PS has a flexible and wellbalanced operator able to enhance and adapt the global as well as fine tune the local search. A helpful review of direct search methods for unconstrained optimization may be found in [13].

The PS algorithm computes a sequence of points that may or may not approach the optimum. First, a set of points called a mesh is established around an existing point (initial or from the previous step). If a point in the mesh is found to improve the objective function, the new point becomes the current point at the next iteration. The PS begins with an initial point $\mathrm{x}_{0}$ supplied by the user. At the first iteration, with a scalar $=1$ (called the mesh size), the pattern vectors (or direction vectors) are constructed as [0 1], [ [ 10$],\left[\begin{array}{ll}-1 & 0\end{array}\right]$ and $\left[\begin{array}{ll}0 & -1\end{array}\right]$, and new mesh points are added as illustrated in Figure 2. Objective functions are computed until a value smaller than for $\mathrm{x}_{0}$ is found. If there is such a point, the poll is successful and the algorithm sets this point as equal to $\mathrm{x}_{1}$. 


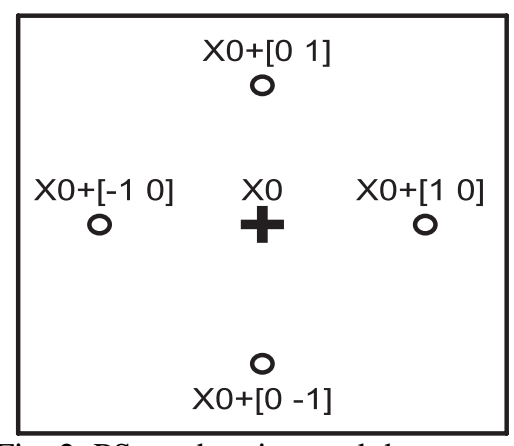

Fig. 2. PS mesh points and the pattern.

After a successful poll, the algorithm multiplies the current mesh size by 2, (called the expansion factor) and proceeds to iteration 2 with the following new points: $2 *\left[\begin{array}{ll}1 & 0\end{array}\right]+\mathrm{x} 1,2 *\left[\begin{array}{ll}0 & 1\end{array}\right]+\mathrm{x} 1,2 *\left[\begin{array}{ll}-1 & 0\end{array}\right]+\mathrm{x} 1$ and $2 *[0-1]+x 1$. If a value smaller than for $x_{1}$ is found, $x_{2}$ is defined, the mesh size is increased by 2 and iterations continue. If at any stage the poll is unsuccessful, i.e. no point has an objective function smaller than the most recent value, the current point is not changed and the mesh size is reduced (e.g. by multiplying by 0.5 , a contraction factor). These steps are repeated until the optimum is found, that is one of the terminating conditions occurs, for example the mesh size is less than the set tolerance, the maximum number of iterations has been reached, the change in the value of the objective function is very small, or similar. The algorithm is depicted in Figure 3.

Constraints need to be handled carefully [14]; in this study they have been augmented to the objective function using Lagrange multipliers, resulting in an increase of the size of the problem through introduction of new parameters [15-18]. For more explanations on how to handle constraints refer to [17, 19, 20].

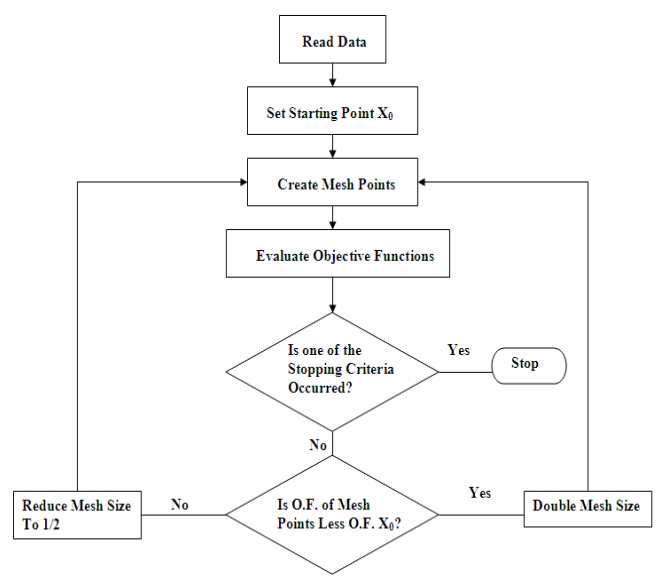

Fig.3: Flow chart of Pattern Search [14].

\section{IV.NUMERICAL RESULTS}

In order to asses the effectiveness and robustness of the proposed hybrid method, several test cases of economic load dispatch with valve point effect have been considered. The non-linear minimization problem formulation of all test cases has been solved using the predefined functions ga, patternsearch and fmincon incorporated into the GA \& DS toolbox of Matlab [19]. Consequently, cost coefficients of the fuel cost and the combined objective function for the considered test cases were coded in Matlab environment.

Initially, several runs were carried out with different values of the key parameters of GA, PS and SQP, such as migration rate, cross-over rate, population size, initial mesh size, and mesh expansion and contraction factors. For the GA, different values for migration rates and cross-over were chosen for each case, whereas the population size for GA was set to 100. The mesh size and the mesh expansion and contraction factors were selected as 1,2 and 0.5 respectively. As for the terminating criteria in PS iterations, all tolerances were set to $10^{-6}$ and the maximum number of iterations and function evaluations were chosen to be 1000 . All runs were conducted using a $3.4 \mathrm{GHz}$ Pentium(R) 2GB of RAM desktop computer, so the comparisons of computing times with those given in literature should be fair.

Case I: Three Generating Units (with losses)

In this case, we considered a test system containing three generators with transmission losses that was reported in [21]. The results of the optimal generation for each generator and the optimal fuel cost are compared with the result of HSS method presented in [22] and those obtained from the conventional method obtained in [21]. Moreover, the generation losses values were also compared with results presented in the literature.

The results of the proposed hybrid method are listed in Table 1. The GA-PS-SQP algorithm has outperformed the other methods in terms of total production cost and power system losses. Moreover, the algorithm has overcome the previous drawback of the need to supply a good initial point in order to reach its global or near global solution.

Table 1: Total Cost and System Losses Comparison

\begin{tabular}{c|cc|c|c}
\hline Generator & $\begin{array}{c}\text { Conventional } \\
\text { Method }\end{array}$ & HSS & PS & GA-PS-SQP \\
\hline $\mathbf{1}$ & 60.2677 & 73.66 & 75.4993 & 77.2881 \\
2 & 79.4462 & 69.98 & 75.0931 & 74.6855 \\
\hline $\mathbf{3}$ & 80.1503 & 75.18 & 67.9488 & 66.4564 \\
\hline Cost $(\$ / \mathbf{h})$ & 3168.623 & 3164.504 & 3160.852 & 3160.774 \\
\hline Losses & 9.865 & 8.820 & 8.541 & 8.429
\end{tabular}

Figures 4 and Fig.5 illustrate the better performance of the proposed method in terms of the reduction of the total power generation cost and the drop in the system losses. 


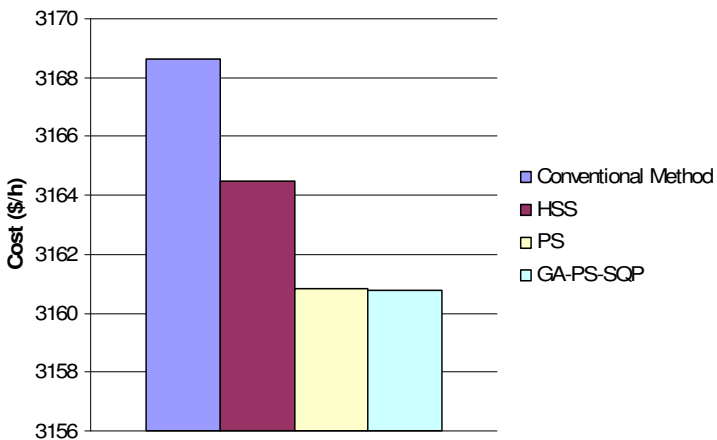

Fig. 4 - Total Cost Comparison Case I

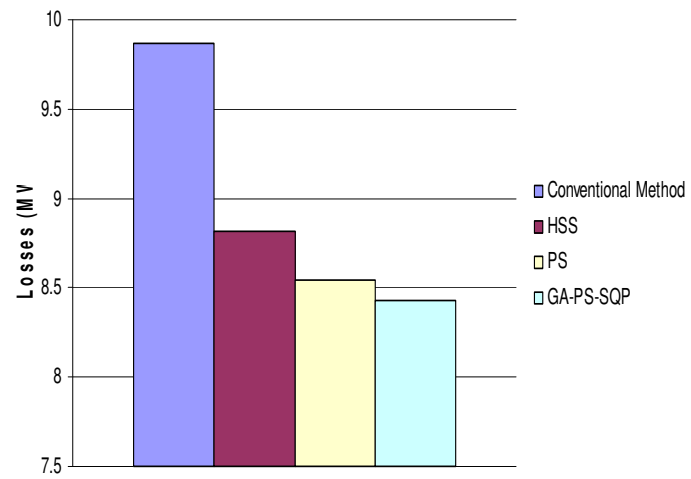

Fig.5 - System Losses Comparison

Case II: Six Generating Units (with emission and losses)

In this combined environmental economic dispatch case, a six-generator system is considered. Information about the generators' fuel cost, NOx emission functions, the $B$ matrix, loss coefficients, and the operating limits are detailed in [23]. The total load demand is set to $700 \mathrm{MW}$, and the weighting factor is 0.5 .

The PS results in terms of the line losses, emission, fuel cost, total cost, and computation time are presented and compared with results of other heuristic methods (Genetic Algorithms, Evolutionary Programming and Pattern Search) from [1, 24, 25] in Table 2. The proposed algorithm has taken more time to reach its optimal solution than the other methods. However, the proposed algorithm has produced the lowest total cost. The nature of the proposed method necessities the increase of computation times, because it runs three methods in sequence.

The total cost of power production and the computation time comparisons between the proposed method and other algorithms in the literature are shown in Figures 6 and 7, respectively

Table 2: Losses, Emission, Total Cost and Computation Time Comparison

\begin{tabular}{|c|c|c|c|c|c|c|}
\hline \multirow{2}{*}{ Generator } & \multicolumn{6}{|c|}{ Optimization Techniques } \\
\hline & IFEP & FEP & CEP & FCGA & PS & $\begin{array}{c}\text { GA-PS- } \\
\text { SQP }\end{array}$ \\
\hline $\mathrm{P} 1(\mathrm{MW})$ & 077.142 & 077.358 & 077.274 & 080.16 & 77.4318 & 74.7285 \\
\hline P2(MW) & 049.925 & 049.669 & 049.639 & 053.71 & 048.894 & 50.9223 \\
\hline P3 (MW) & 048.764 & 048.310 & 048.535 & 040.93 & 048.510 & 49.1058 \\
\hline P4 (MW) & $1 \cap 3486$ & 1104369 & 10352.5 & 11623 & 1045679 & 11744459 \\
\hline $\mathrm{P} 5(\mathrm{MW})$ & 259.805 & 260.663 & 260.695 & 251.20 & 260.8632 & 263.2332 \\
\hline $\mathrm{P} 6(\mathrm{MW})$ & 191.828 & 190.473 & 191.233 & 190.62 & 190.6723 & 188.7740 \\
\hline Line losses (MW) & 30.949 & 30.849 & 30.901 & 32.850 & 30.945 & 31.2108 \\
\hline Enissiun(ky/ll) & 530.5164 & 532.5046 & 524.49 & 527.40 & 528.33 & 529.3694 \\
\hline Fuel cost $(\$ / h)$ & 38214.02 & 38214.23 & 38216.47 & 38408.82 & 38208.63 & 38203.36 \\
\hline Total cost $(\$ / h)$ & 19369.84 & 19369.89 & 19369.84 & 19468.14 & 19368.48 & 19366.36 \\
\hline omputation time (s) & 3.874 & 1.598 & 4.48 & $\cdot$ & 2.05 & 26.67 \\
\hline No. of iteration & 57 & 65 & 77 & - & 40 & - \\
\hline
\end{tabular}

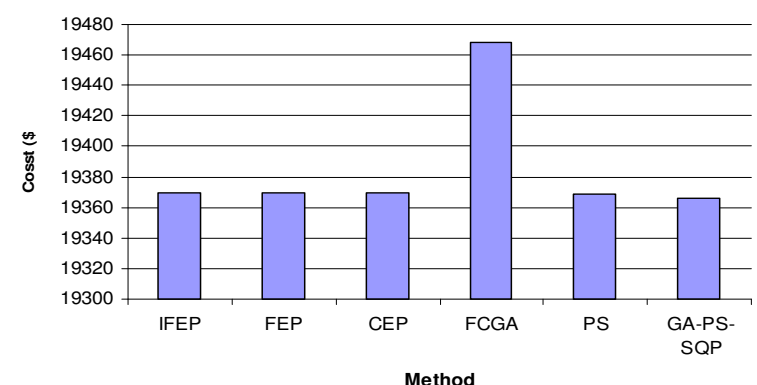

Fig.6 - Total Cost Comparison Case II

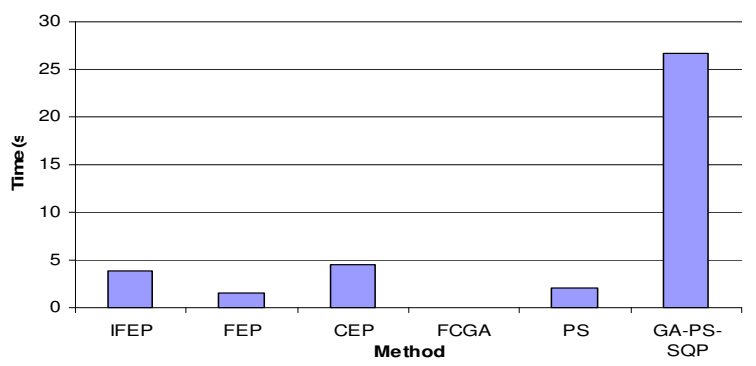

Fig. 7 - Computation Time Comparison Case II

\section{Case III: Multi Area Economic Dispatch (MAED)}

The multi area ED problem considered consists of four areas with tie lines connecting these areas. Each area contains four generation units. Note that quadratic cost functions are used to model the cost of generation $\mathrm{F}\left(\mathrm{P}_{\mathrm{gi}}\right)$, but the tie lines transmission costs, $G\left(T_{j k}\right)$, are assumed to be linear functions of the power transfer. The generators' data and the tie lines coefficients along with their limits are all given in reference [26].

Different heuristic methods such as PSO, EP and PS have been applied to the same problem and results reported in [1, 24, 25]. The results using the PS and the other methods are shown in Tables 3 and 4. Like in the previous, case the proposed algorithm has taken more time to solve and, since this case is more complicated in nature than Case II, the large difference in computation times is clearly noticeable. 
Table 3: Generators Productions

\section{Optimization techuiqu ues}

\begin{tabular}{|c|c|c|c|c|c|c|c|}
\hline \multicolumn{2}{|c|}{ Geherator } & \multirow[b]{2}{*}{ IFEP } & \multirow[b]{2}{*}{ FEP } & \multirow[b]{2}{*}{ CEP } & \multirow[b]{2}{*}{ NFP } & \multicolumn{2}{|r|}{ GA-PS- } \\
\hline & & & & & & PS & SOP \\
\hline \multirow{4}{*}{$\begin{array}{l}\text { Area l } \\
\text { Demand } \\
400 \mathrm{MW}\end{array}$} & $\mathrm{Pl}(\mathrm{MW})$ & 149.998 & 149097 & 150,0000 & 150.000 & 1500000 & 150,000 \\
\hline & $\mathrm{F} 2(\mathrm{MW})$ & 099.986 & 099968 & 10000000 & 100.000 & 1000000 & 1000000 \\
\hline & $\mathrm{B} 3(\mathrm{MW})$ & 0168.270 & 067.017 & 068826 & 066.970 & 066971 & 670078 \\
\hline & $P 4(\mathrm{MW})$ & 0909.940 & 009.774 & 099,98 & 100.000 & 1000000 & 10000000 \\
\hline \multirow{4}{*}{$\begin{array}{l}\text { Area 2 } \\
\text { Demand } \\
200 \mathrm{WW}\end{array}$} & $\mathrm{Fs}(\mathrm{MMM})$ & 056.349 & 057.181 & 056373 & 056970 & 56.9718 & 5700085 \\
\hline & $\mathbb{P}(\mathrm{MW})$ & 0196.753 & 019554 & 093519 & 096.250 & 96.2518 & 96.2605 \\
\hline & $F(M M)$ & 041.264 & 041.736 & 042546 & 041.870 & 41.8718 & 418786 \\
\hline & $\mathrm{PB}(\mathrm{MW})$ & 072.586 & 072.748 & 072647 & 072.520 & 72.5218 & 72.5070 \\
\hline \multirow{4}{*}{$\begin{array}{l}\text { Area } 3 \\
\text { Demand } \\
350 \mathrm{WW}\end{array}$} & $P(\mathrm{MW})$ & 0150.003 & 0150.030 & 050.010010 & 050.000 & 050.002 & 50.01000 \\
\hline & $\mathrm{F} 10(\mathrm{MW})$ & 0135.985 & 013652 & 036399 & 036.270 & 0136.272 & 36.2541 \\
\hline & $\mathrm{P} 11(\mathrm{MW})$ & 038.012 & 038413 & 03832 & 038.490 & 038.492 & 385041 \\
\hline & P12(MW) & 037.426 & 037001 & 0369013 & 037320 & 037.322 & 373112 \\
\hline \multirow{4}{*}{$\begin{array}{l}\text { Area } 4 \\
\text { Demand } \\
300 \mathrm{WW}\end{array}$} & $\mathrm{P} 13(\mathrm{MW})$ & 149.988 & 149.986 & 150.0000 & 150.000 & 1500000 & 150.0000 \\
\hline & Pl4 (MW) & 099.964 & 010995 & 100.01000 & 1000000 & 1000000 & 10000000 \\
\hline & P1S (MW) & 057.601 & 0157.568 & 056648 & 057.050 & 0157.051 & 5701081 \\
\hline & $P 16(\mathbb{M W})$ & 095.874 & 096.482 & 095866 & 096.270 & 096.271 & 962600 \\
\hline
\end{tabular}

Table 4: Tie Lines and Total Cost Comparison

\begin{tabular}{|c|c|c|c|c|c|c|c|}
\hline \multicolumn{2}{|c|}{ Area } & \multicolumn{6}{|c|}{ Tie lines values (M W) } \\
\hline From & To & IFEP & FEP & CEP & NFP & PS & $\begin{array}{c}\text { GA-PS } \\
\text { SOP }\end{array}$ \\
\hline 1 & 2 & 010094 & 010.062 & 00.000 & 010.0010 & 010000 & 00.000 \\
\hline 1 & 3 & 18649 & 18.241 & 19.87 & 18.180 & 18.181 & 20.276 \\
\hline 1 & 4 & 00000 & 010.0000 & 000000 & 010.0000 & 00000 & 00.000 \\
\hline 2 & 1 & 010.018 & 010.00010 & 00.018 & 010.0000 & 000000 & 000000 \\
\hline 2 & 3 & 69997 & 69.790 & 68.861 & 69.730 & 69.730 & 67.655 \\
\hline 2 & 4 & 101000 & 010.0010 & 00.0010 & 010.0010 & 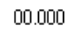 & 00.0100 \\
\hline 3 & 1 & 000000 & 010.000 & 000000 & 010.000 & 000000 & 00.000 \\
\hline 3 & 2 & 00000 & [10.0010 & 000000 & [10.0010 & 00000 & 00.000 \\
\hline 3 & 4 & 010000 & 010.0010 & 00.0010 & 0000010 & 000000 & 00.0100 \\
\hline 4 & 1 & 010.549 & 01.548 & 00.758 & 01.210 & 01.210 & 3.268 \\
\hline 4 & 2 & 02.951 & 02.509 & 01.789 & 02.110 & 02.111 & 00.0100 \\
\hline 4 & 3 & 99.927 & 99.974 & 99.927 & 100.00 & 100.00 & 100.00 \\
\hline \multicolumn{2}{|c|}{ Total $\cos (\$ / h)$} & 7337.51 & 7337.52 & 7337.75 & 733700 & 733698 & 7337.01 \\
\hline \multicolumn{2}{|c|}{ Computation time(s) } & 23.97 & 7.47 & 78211.49 & - & 5.77 & 61.25 \\
\hline \multicolumn{2}{|c|}{ Population size } & 100 & 100 & 100 & - & - & 100 \\
\hline \multicolumn{2}{|c|}{ No of ite rations } & 585 & 645 & 758920 & - & 1225 & - \\
\hline
\end{tabular}

Figure 8 shows the competitiveness of the proposed method with NFP and PS in terms of reduction of the total cost of the four areas generation production. The comparison of the computation times is illustrated in Figure 9.

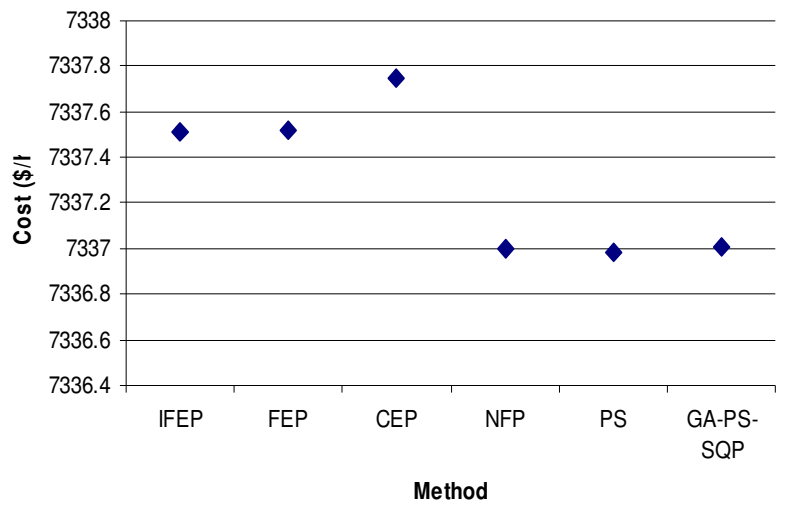

Fig. 8 - Total Cost Comparison Case III

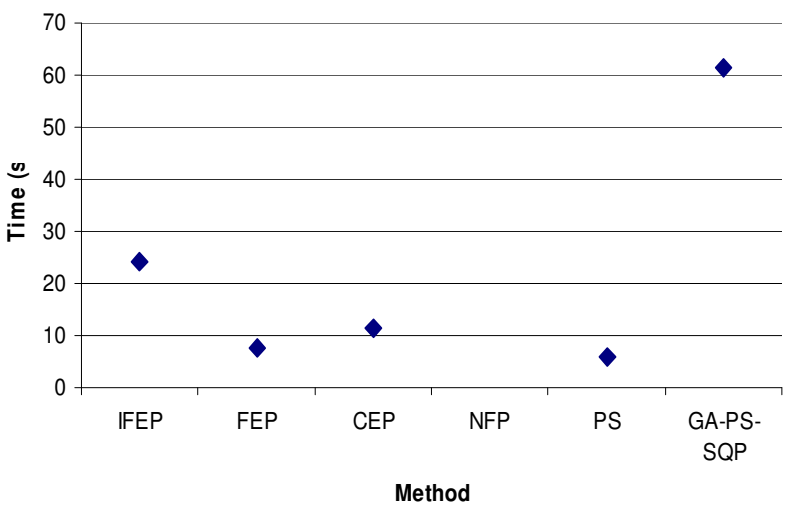

Fig.9 - Computation Time Comparison Case III

It should be noted that the proposed hybrid method produced the final result in a single run; whereas PS needed 100 runs to find a proper starting point that led to its solution. As a result the total execution time for the PS method (402 s in case II and $1473.6 \mathrm{~s}$ in case III) should be considered as the sum of the 100 runs. In this case, the comparison of the execution times shows that the proposed hybrid method has led to a significant saving in computation time.

\section{CONCLUSION}

A new hybrid method based on a combination of Genetic Algorithm (GA), Pattern Search (PS) and Sequential Quadratic Programming optimization (SQP) to solve several cases of the economic dispatch problem is presented. A comparison with other methods from literature in terms of total power generation cost, system losses and computation time was conducted and discussed. The proposed algorithm has outperformed the other methods in terms of achieving lower total generation cost and system losses. The penalty is that the proposed algorithm requires an extended computation time to reach the solution due to the execution of three different methods sequentially. However, the GA-PS-SQP technique has overcome an important drawback of the PS or SQP methods that is the need to supply a suitable starting point. This shortcoming of the PS and SQP methods was highlighted before; it makes any optimization method relying on a good choice of the initial point possibly more susceptible to getting trapped in local minima. The hybrid GA-PS-SQP algorithm, on the other hand, does not require the user to specify the starting point as it is generated automatically for the final SQP stage by the initial GA-PS search. Moreover, the comparison with the total execution time between the proposed hybrid method and PS has led to a massive saving in computation time. 


\section{REFERENCES}

[1] J. S. Al-Sumait, J. K. Sykulski, and A. K. Al-Othman, "Solution of different types of economic load dispatch problems using a pattern search method," Electric Power Components and Systems, vol. 36, pp. 250-265, 2008.

[2] J. S. Al-Sumait, A. K. Al-Othman, and J. K. Sykulski, "Application of pattern search method to power system valve-point economic load dispatch," International Journal of Electrical Power and Energy Systems, vol. 29, pp. 720-730, 2007.

[3] C. L. Chen and N. Chen, "Direct search method for solving economic dispatch problem considering transmission capacity constraints," IEEE Transactions on Power Systems, vol. 16, pp. 764-769, 2001.

[4] E. Tsoi, K. P. Wong, and C. C. Fung, "Hybrid GA/SA algorithms for evaluating trade-off between economic cost and environmental impact in generation dispatch," Perth, Aust, 1995, pp. 132-137.

[5] N. Thenmozhi and D. Mary, "Economic emission load dispatch using hybrid Genetic Algorithm," Chiang Mai, Thailand, 2004, pp. 476-479.

[6] T. A. A. Victoire and A. E. Jeyakumar, "Hybrid PSOSQP for economic dispatch with valve-point effect," Electric Power Systems Research, vol. 71, pp. 51-59, 2004.

[7] L. dos Santos Coelho and V. C. Mariani, "Combining of chaotic differential evolution and quadratic programming for economic dispatch optimization with valve-point effect," IEEE Transactions on Power Systems, vol. 21, pp. 989-996, 2006.

[8] T. A. A. Victoire and A. E. Jeyakumar, "A tabu search based hybrid optimization approach for a fuzzy modelled unit commitment problem," Electric Power Systems Research, vol. 76, pp. 413-425, 2006.

[9] J. O. Kim, D.-J. Shin, J.-N. Park, and C. Singh, "Atavistic genetic algorithm for economic dispatch with valve point effect," Electric Power Systems Research, vol. 62, pp. 201-207, 2002.

[10] D. C. Walters and G. B. Sheble, "Genetic algorithm solution of economic dispatch with valve point loading," Power Systems, IEEE Transactions on, vol. 8, pp. 1325-1332, 1993.

[11] D. E. Goldberg, Genetic algorithms in search, optimization, and machine learning. Reading, Mass. ; Harlow: Addison-Wesley, 1989.

[12] Z. Michalewicz, Genetic algorithms + data structures = evolution programs, 3rd rev. and extended ed. Berlin ; New York: Springer-Verlag, 1996.

[13] R. M. Lewis, V. Torczon, and M. W. Trosset, "Direct search methods: then and now," Journal of Computational and Applied Mathematics, vol. 124, pp. 191-207, 2000.

[14] H. K. Youssef, M.El-Shibini, and G.A.Hazaa, "Some new aspects in power system dynamic security using pattern recognition," in Second Middle East Power Conference. MEPCON-92, 1992, pp. 308-313.

[15] R. M. Lewis and V. Torczon, "Pattern search algorithms for linearly constrained minimization," SIAM Journal on Optimization, vol. 10, pp. 917-941, 1999.

[16] R. M. Lewis and V. Torczon, "Pattern search algorithms for bound constrained minimization," SIAM Journal on Optimization, vol. 9, pp. 1082-99, 1999.
[17] R. M. Lewis and V. Torczon, "A globally convergent augmented Lagrangian pattern search algorithm for optimization with general constraints and simple bounds," SIAM Journal on Optimization, vol. 12, pp. 1075-89, 2001.

[18] V. Torczon, "On the convergence of pattern search algorithms," SIAM Journal on Optimization, vol. 7, pp. $1-25,1997$.

[19] "Genetic Algorithm and Direct Search Toolbox for use with Matlab user's guide," 2 ed: The Math works inc.

[20] A. R. Conn, N. I. M. Gould, and P. L. Toint, "A Globally convergent augmented Lagrangian algorithm for optimization with general constraints and simple bounds," SIAM Journal on Numerical Analysis, vol. 28, pp. 545-572, 1991.

[21] N. Sinha, R. Chakrabarti, and P. K. Chattopadhyay, "Evolutionary programming techniques for economic load dispatch," Evolutionary Computation, IEEE Transactions on, vol. 7, pp. 83-94, 2003.

[22] D. Bhagwan Das and C. Patvardhan, "Solution of Economic Load Dispatch using real coded Hybrid Stochastic Search," International Journal of Electrical Power \& Energy Systems, vol. 21, pp. 165-170, 1999.

[23] J. S. Dhillon, S. C. Parti, and D. P. Kothari, "Stochastic economic emission load dispatch," Electric Power Systems Research, vol. 26, pp. 179-186, 1993.

[24] T. Jayabarathi, K. Jayaprakash, D. N. Jeyakumar, and T. Raghunathan, "Evolutionary programming techniques for different kinds of economic dispatch problems," Electric Power Systems Research, vol. 73, pp. 169-176, 2005.

[25] D. N. Jeyakumar, T. Jayabarathi, and T. Raghunathan, "Particle swarm optimization for various types of economic dispatch problems," International Journal of Electrical Power \& Energy Systems, vol. 28, pp. 36-42, 2006.

[26] D. Streiffert, "Multi-area economic dispatch with tie line constraints," Power Systems, IEEE Transactions on, vol. 10, pp. 1946-1951, 1995.

Jamal S. Alsumait received the B.S from the University of Miami in 1990, and M.S. from Kuwait University in 2003.

$\mathrm{He}$ is currently working toward the PhD degree in the Electrical Power Engineering Research Group at Southampton University.

Jan K. Sykulski is a Professor of Applied Electromagnetics and Head of Electrical Power Engineering Group of the School of Electronics and Computer Science at the University of Southampton. His research interests are in the areas of power applications of high temperature superconductivity, modelling of superconducting materials, advances in simulations of coupled field systems, development of fundamental methods of computational electromagnetics and new concepts in design and optimization of electromechanical devices. He has 283 publications listed on the official database of the University. $\mathrm{He}$ is a founding Secretary/Treasurer/Editor of International Compumag society, a Visiting Professor at universities in Canada, Italy, Poland, France and China, a founding member of International Steering Committees of major international conferences, including COMPUMAG, IEEE CEFC, IEE CEM, ISEF, EPNC and others. He is Fellow of IEE, Institute of Physics and British Computer Society. 